\title{
Discrete Dynamics in Evolutionary Computation and Its Applications
}

\author{
Yong-Hyuk Kim, ${ }^{1}$ Ahmed Kattan, ${ }^{2}$ Michael Kampouridis, ${ }^{3}$ and Yourim Yoon ${ }^{4}$ \\ ${ }^{1}$ Department of Computer Science and Engineering, Kwangwoon University, 20 Kwangwoon-ro, Nowon-gu, \\ Seoul 01890, Republic of Korea \\ ${ }^{2}$ EvoSys Ltd., King's Road Tower, Level 26, Jeddah 21499, Saudi Arabia \\ ${ }^{3}$ School of Computing, University of Kent, Medway Campus, Chatham Maritime, Kent ME4 4AG, UK \\ ${ }^{4}$ Department of Computer Engineering, Gachon University, 1342 Sengnamdaero, Sujeong-gu, Seongnam-si, \\ Gyeonggi-do 13120, Republic of Korea \\ Correspondence should be addressed to Yourim Yoon; yryoon@gachon.ac.kr
}

Received 31 May 2016; Accepted 31 May 2016

Copyright (C) 2016 Yong-Hyuk Kim et al. This is an open access article distributed under the Creative Commons Attribution License, which permits unrestricted use, distribution, and reproduction in any medium, provided the original work is properly cited.

Evolutionary computation (EC) is considered to be a natural and artificial system with discrete dynamics. Over the last few decades, there has been a remarkable growth in the field of EC, encompassing large interest and efforts from researchers. EC has been successfully applied to various realworld problems for optimization purposes. The aim of this special issue is to publish original and high-quality articles related to discrete dynamics in EC and its applications.

This special issue was opened in November of 2015 and closed in February of 2016. There were a total of 29 submissions. All of them were peer-reviewed according to the high standards of this journal and only 5 of them were accepted for publication, which gave important developments in discrete dynamics in EC and its applications. Among the accepted papers, one is for the discrete dynamics in EC and the others are for two major applications of EC, operations research and network problems. The guest editors of this special issue hope that the presented results could outline new ideas for further studies.

In EC, selection or mating is one of the most important operations. In the paper entitled "A New Adaptive Hungarian Mating Scheme in Genetic Algorithms," C. Jung et al. suggested an adaptive mating scheme from Hungarian mating schemes, which consist of maximizing the sum of mating distances, minimizing the sum, and random matching. They presented an adaptive algorithm to elect one of these Hungarian mating schemes. Each mated pair of individuals voted for the next generation mating scheme. The distance between parents and the distance between parent and offspring were considered during voting. Two wellknown combinatorial optimization problems, the traveling salesman problem and the graph bisection problem, which are NP-hard, were considered to show the effectiveness of their adaptive method.

In the paper entitled "A Hybrid IP/GA Approach to the Parallel Production Lines Scheduling Problem," H. Ren and S. Sun studied a scheduling problem of parallel production lines. Considering the time window and some technical constraints, they formulated a mixed integer programming model for the problem. They also deduced some valid inequalities and presented a hybrid mixed integer programming/constraint programming decomposition strategy. Based on them, the authors proposed a hybrid genetic algorithm for efficiently solving the problem.

In the paper entitled "Solving a Closed-Loop LocationInventory-Routing Problem with Mixed Quality Defects Returns in E-Commerce by Hybrid Ant Colony Optimization Algorithm," S. Deng et al. presented a closed-loop locationinventory-routing problem model considering both quality defect returns and nondefect ones in e-commerce supply chain system, to minimize the total cost produced in both forward and reverse logistics networks. They proposed a hybrid ant colony optimization algorithm for efficiently solving this model that is NP-hard. 
Since various factors affect the fluctuation of network traffic, accurate prediction of network traffic is considered as a challenging task of the time series prediction process. In the paper entitled "A Network Traffic Prediction Model Based on Quantum-Behaved Particle Swarm Optimization Algorithm and Fuzzy Wavelet Neural Network," K. Zhang et al. proposed a novel prediction method of network traffic based on quantum-behaved particle swarm optimization (QPSO) algorithm and fuzzy wavelet neural network (FWNN). The authors introduced QPSO and presented the structure and operation algorithms of FWNN. The parameters of FWNN were optimized by a QPSO algorithm. This optimized QPSOFWNN was applied to the prediction of network traffic successfully when compared to different prediction models such as BP neural network, RBF neural network, fuzzy neural network, and FWNN-GA neural network.

The exponential growth in data traffic due to the modernization of smart devices has resulted in the need for a high-capacity wireless network in the future. To successfully deploy $5 \mathrm{G}$ networks, we should be able to handle the growth in the data traffic. The increasing amount of traffic volume puts excessive stress on the important factors of the resource allocation methods such as scalability and throughput. In the paper entitled "A Genetic Algorithm with Location Intelligence Method for Energy Optimization in 5G Wireless Networks," R. Sachan et al. defined network planning as an optimization problem with the decision variables such as transmission power and transmitter location in 5G networks, leading to interesting implementation using some heuristic approaches such as differential evolution and realcoded genetic algorithm (RCGA). The authors modified an RCGA-based method to find the optimal configuration of transmitters by not only offering optimal coverage of underutilized transmitters, but also optimizing the amounts of power consumption.

In sum, the five papers present some of the latest and most promising research results on EC and its applications. This special issue demonstrates the theoretical and practical importance of further studies on EC. The guest editors of this special issue hope that these papers may enrich and provide a guide to the readers to treat EC or further developments in the applications of EC.

\section{Acknowledgments}

The guest editors of this special issue would like to express their gratitude to all of the authors for their contributions and the reviewers for their effort providing helpful comments and suggestions. This work was supported by the Gachon University Research Fund of 2015 (GCU-2015-0030) and a grant [MPSS-CG-2016-05] through the Disaster and Safety Management Institute funded by the Ministry of Public Safety and Security of the Korean government.

Yong-Hyuk Kim Ahmed Kattan

Michael Kampouridis Yourim Yoon 


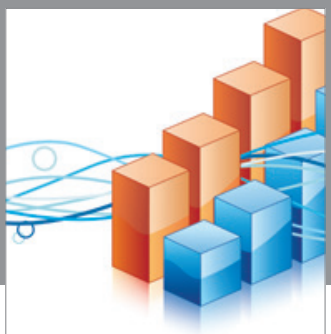

Advances in

Operations Research

vatem alat4

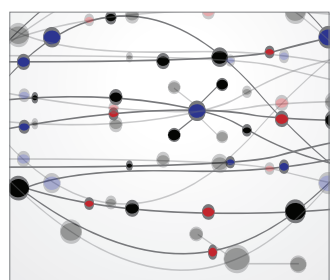

\section{The Scientific} World Journal
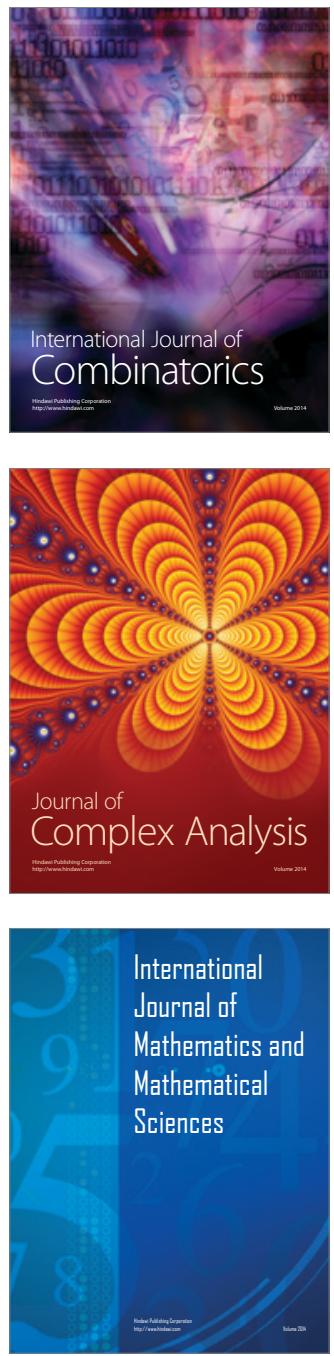
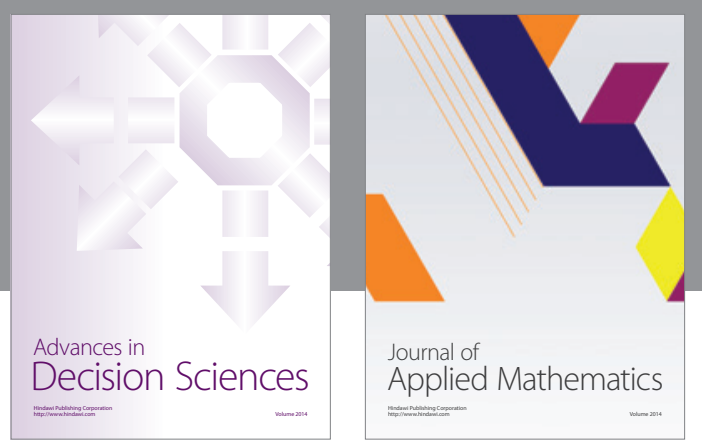

Algebra

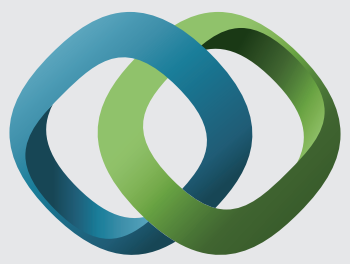

\section{Hindawi}

Submit your manuscripts at

http://www.hindawi.com
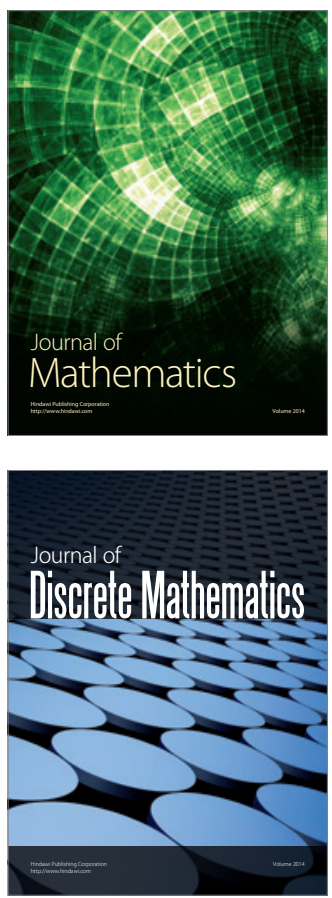

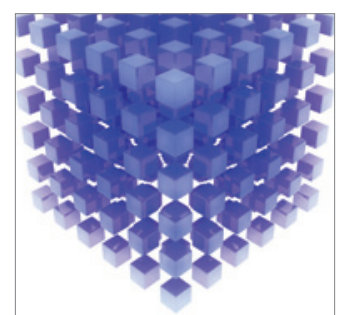

Mathematical Problems in Engineering
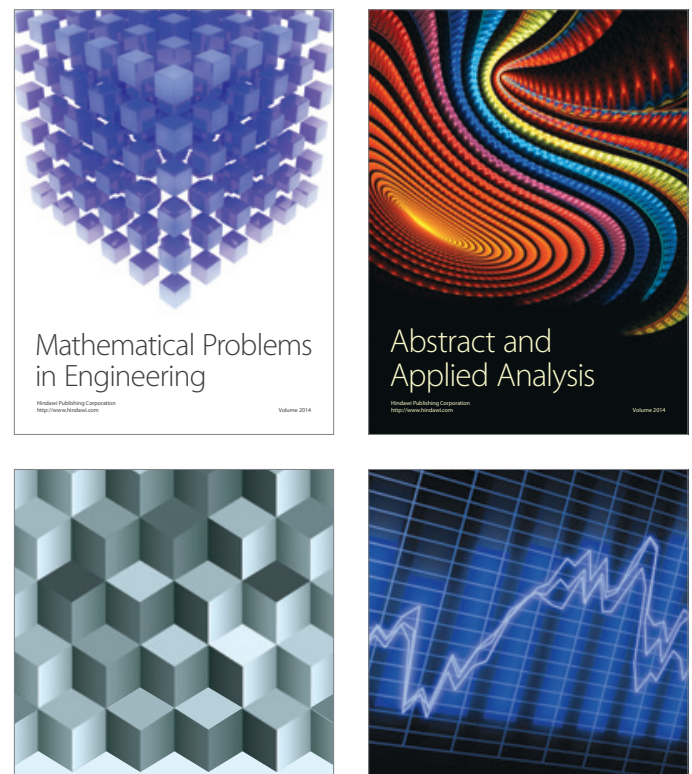

Journal of

Function Spaces

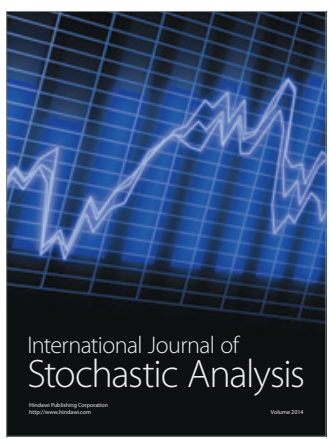

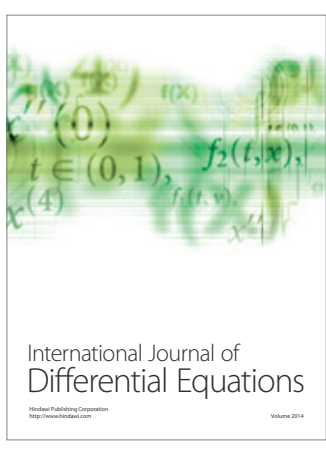
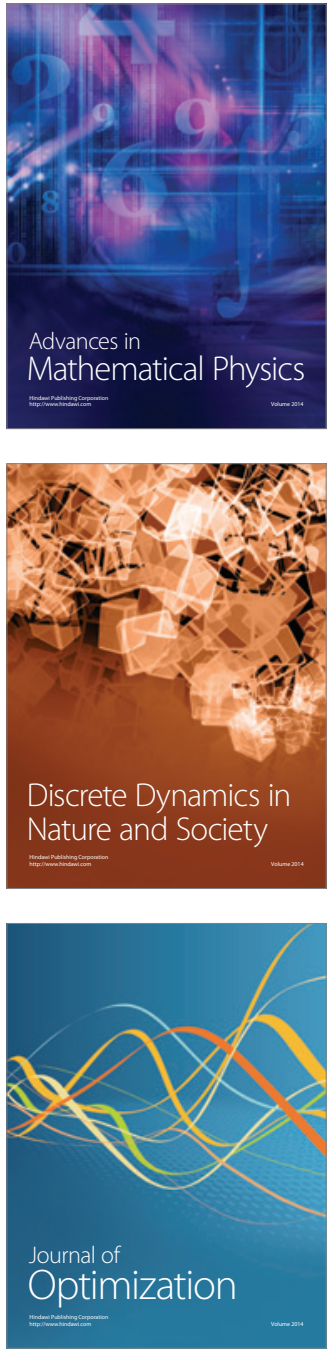